\title{
BMJ Open Gestational diabetes mellitus, pre- gestational BMI and offspring BMI z-score during infancy and childhood: 2004 Pelotas Birth Cohort
}

\author{
Romina Buffarini, ${ }^{1}$ Aluisio J D Barros, ${ }^{1}$ Alicia Matijasevich, ${ }^{2}$ \\ Christian Loret de Mola, ${ }^{3}$ Ina S Santos ${ }^{1}$
}

To cite: Buffarini R, Barros AJD, Matijasevich A, et al. Gestational diabetes mellitus, pre-gestational BMI and offspring BMI z-score during infancy and childhood: 2004 Pelotas Birth Cohort. BMJ Open 2019;9:e024734. doi:10.1136/ bmjopen-2018-024734

- Prepublication history and additional material for this paper are available online. To view these files, please visit the journal online (http://dx.doi. org/10.1136/bmjopen-2018024734).

Received 18 June 2018 Revised 13 March 2019 Accepted 7 June 2019

Check for updates

(c) Author(s) (or their employer(s)) 2019. Re-use permitted under CC BY-NC. No commercial re-use. See rights and permissions. Published by BMJ.

${ }^{1}$ Postgraduate Program in Epidemiology, Federal University of Pelotas (UFPel), Pelotas, Rio Grande do Sul, Brazil

${ }^{2}$ Faculdade de Medicina, Universidade de Sao Paulo, Sao Paulo, Brazil

${ }^{3}$ Postgraduate Program in Public Health, Federal University of Rio Grande, Rio Grande, Brazil

Correspondence to Dr Romina Buffarini; romibuffarini@gmail.com

\section{ABSTRACT}

Objective Gestational diabetes mellitus (GDM) affects a significant number of women. Evidence regarding the association between GDM and offspring body mass index (BMI) is unclear due to small samples and lack of adequate confounding control. The objective of this study was to investigate the association between GDM and offspring $\mathrm{BMI} z$-scores from birth to early adolescence and to examine the role of maternal pre-gestational BMI in this relationship.

Design Prospective study.

Setting Pelotas 2004 Birth Cohort, Brazil.

Participants Cohort participants that were followed-up from birth up to early adolescence ( 3500) and their mothers.

Primary outcome measures BMI z-scores at birth, 3 , $12,24,48$ months and 6 and 11 years of age, calculated according to the WHO growth charts.

Results Unadjusted and adjusted linear regressions were performed and interaction terms between maternal pregestational BMI and GDM were included. Prevalence of self-reported GDM was $2.6 \%(95 \% \mathrm{Cl} 2.1 \%$ to $3.1 \%)$. The offspring BMI z-scores (SD) at birth, 3, 12, 24, 48 months and at 6 and 11 years were $0.10(1.12),-0.47(1.10)$, 0.59 (1.10), 0.59 (1.08), 0.78 (1.32), 0.70 (1.43) and 0.75 (1.41), respectively. Unadjusted regression models showed positive associations between GDM and offspring BMI z-scores at birth, 6 and 11 years. After adjustment, the associations attenuated towards the null. Statistical evidence of effect modification between maternal pregestational BMl and GDM was observed at birth $(p=0.007)$, with the association between GDM and offspring BMI z-score being apparent only in those children born to overweight or obese mothers $(\beta=0.72,95 \% \mathrm{Cl} 0.30$ to 1.14 and $\beta=0.61,95 \% \mathrm{Cl} 0.20$ to 1.01 , respectively). Conclusions We observed that in the association between GDM and offspring BMI z-scores, there is a predominant role for maternal nutritional status before pregnancy and that the association between GDM and newborn's BMI is apparent only among those born to overweight or obese mothers.

\section{INTRODUCTION}

Gestational diabetes mellitus (GDM), defined as any degree of glucose intolerance with
Strengths and limitations of this study

- Population-based birth cohort with high follow-up rates.

- Availability of anthropometric measurements collected prospectively over 11 years since birth performed by trained anthropometrists.

- Inclusion of maternal pre-gestational body mass index (BMI) as a confounder or effect modifier for the association between gestational diabetes mellitus (GDM) and offspring BMI z-scores.

Distinction between GDM and existing diabetes.

- Limitations of our study are the lack of data on glycaemic control during pregnancy and the assessment of GDM based on maternal self-reporting, which could lead to misclassification.

onset or first recognition during pregnancy, ${ }^{1}$ affects between $2 \%$ and $14 \%$ of pregnancies, with considerable variation related to differences in diagnosis criteria, geographical and ethnic characteristics. ${ }^{2-4}$ Its prevalence seems to be growing globally. ${ }^{5}$

In addition to the well-documented shortterm adverse outcomes on the fetus (macrosomia, neonatal hypoglycaemia, respiratory distress syndrome, shoulder dystocia, among others), ${ }^{67}$ it has been suggested that GDM can lead to offspring long-term health impacts. The hypothesised biological mechanism is that exposure to a hyperglycaemic environment in utero could influence fetal development and lead to an increased risk of becoming obese later in life (developmental overnutrition theory). Potential pathways linking hyperglycaemia and long-term adverse cardiometabolic outcomes include greater insulin secretion, which, in turn, increases fetal fat deposition, epigenetic modification (DNA methylation) and differential programming of tissues and organs as pancreas and hypothalamic-endocrine 
system. Other hypotheses involve genetic inheritance and environmental factors. ${ }^{89}$

Obesity, along with overweight, affects over a third of the world's population today and the prevalence in the $5-19$ years age range is rising rapidly. ${ }^{10} 11$ Thus, there is a growing concern about childhood overweight and obesity, as they are strongly associated with chronic disease morbidity and mortality later in life. ${ }^{1213}$

In the recent years, several epidemiologic studies have assessed the influence of diabetes in pregnancy (gestational onset, type I or type II) on offspring body mass index (BMI) and obesity at different ages during childhood. ${ }^{14-26}$ However, some of these studies contained a mixture of GDM with existing diabetes before pregnancy, ${ }^{18192324}$ were limited by small sample size ${ }^{1521-24}$ or very specific populations (eg, Pima Indian Study, hospital-based sample and Mexican American children) ${ }^{21} 2526$ and most of them did not adequately accounted for important confounders (eg, pre-gestational maternal BMI), ${ }^{14} 151722-24$ thus yielding controversial results.

A meta-analysis of observational studies about diabetes during pregnancy and offspring BMI z-scores revealed that only three of a total of nine studies had controlled for maternal BMI. ${ }^{27}$ Of these, one found independent association between prenatal exposure to diabetes and children greater BMI at age 7 years, ${ }^{19}$ and the two others did not find any association at ages 3 and 9-11 years, respectively. ${ }^{16} 18$ Therefore, our study may contribute to the literature regarding the role of maternal BMI before pregnancy in the association between GDM and offspring BMI.

This study was aimed at assessing the association between GDM and offspring's BMI z-score from birth to 11 years of age in a Southern Brazilian population that have been prospectively followed since birth. We also explored the role of maternal pre-gestational BMI as a confounder or effect modifier in the association between GDM and offspring BMI z-score. Our hypothesis was that children born to mothers with GDM would have higher BMI z-scores means than children born to mothers without GDM, and that the association between GDM and offspring BMI z-score would remain after adjustment for maternal pre-gestational BMI.

\section{METHODS}

In 2004, five maternity hospitals in Pelotas, southern Brazil, were visited daily and all births were identified. Live newborns (99.2\% of deliveries) whose families lived in the urban area of the city were examined and their mothers were interviewed within 24 hours of delivery. The full cohort has been followed up at 3, 12, 24, 48 months and at 6 and 11 years of age, with $95.7 \%, 94.3 \%$, $93.5 \%, 92.0 \%, 90.2 \%$ and $86.6 \%$ response rates, respectively. Demographic, socioeconomic, behavioural, health status and anthropometric variables were collected at each follow-up. On each occasion, standardised questionnaires were applied by trained interviewers. Of the 4231 participants constituting the original cohort, 98 died in the first eleven years of life. Because the results did not change when replicating all regression models after the exclusion of preterm births, preterm deliveries from mothers with GDM $(n=15)$ and without GDM $(n=590)$ were not excluded from the analyses. Multiple births accounting for 84 newborns were excluded from the current analyses because mean birth weight and growth of twin children differ from birth weight and growth of otherwise similar children but born to single pregnancies. Mothers who already had diabetes before pregnancy $(n=14)$ were also excluded. A flow chart of the 2004 Pelotas Birth Cohort explaining the numbers of interviewed participants and those included in the current study on every phase of the study is showed in online supplementary figure 1 . Detailed information of the cohort procedures at each follow-up is given elsewhere. ${ }^{28-30}$

\section{Exposure variable}

GDM was self-reported by the mother during the perinatal interview, based on the following questions: 'Did you have diabetes or high blood sugar during pregnancy? If yes: 'Did you already have diabetes before pregnancy? Those mothers who already had diabetes mellitus before pregnancy $(n=14)$ were not considered in our study. The self-reported GDM validity had been previously tested in a sample of women in the immediate postpartum. ${ }^{31}$ In that study, prevalence (95\% CI) of GDM as based on the antenatal care card records (gold standard) was 4.3\% (95\% CI $3.0 \%$ to $5.8 \%$ ), while the self-reported rate was $4.0 \%$ (95\% CI $2.8 \%$ to $5.5 \%)$. The study showed that maternal self-reported GDM had $72.9 \%$ (95\% CI: $55.9 \%$ to $86.2 \%$ ) sensitivity, $99.0 \%$ (95\% CI $98.1 \%$ to $99.6 \%$ ) specificity, $97.9 \%$ (95\% CI $96.7 \%$ to $98.7 \%$ ) accuracy and a kappa statistics of $74.0 \% .^{31}$

\section{Outcomes}

Children's anthropometric measurements were collected at birth and at each subsequent follow-up. At the perinatal phase, newborns were weighed by the hospital staff using digital paediatric scales with $10 \mathrm{~g}$ precision, calibrated weekly to standard weights. Birth length was measured by the research cohort team using AHRTAG portable infantometres with $1 \mathrm{~mm}$ precision (AHRTAG, London), custom built for this study. The measurement was carried out in the recumbent position, from top of the head to the heel of one foot. At 3, 12, 24 and 48 months, the interviews and examinations were carried out at home. Supine length ( $\leq 24$ months of age) was taken using the same portable infantometer used in the perinatal phase. At the 4-year visit, height was measured using a portable stadiometer with $1 \mathrm{~mm}$ precision, which was developed for this study. At 6 and 11 years of age the visits took place at the clinic research centre. Weight was measured with a digital scale (Tanita BC-558 Ironman Segmental Body Composition Monitor, maximum $150 \mathrm{~kg}$ and $100 \mathrm{~g}$ precision) and height was taken with a stadiometre (Harpenden) (maximum $2.06 \mathrm{~m}$ and $1 \mathrm{~mm}$ precision). ${ }^{29}$ 
On each occasion, the measurements were performed by trained anthropometrists with the children dressed in underwear and barefoot. When clothing was worn, these items were noted and had their weights subsequently deducted from the child's measured weight. For participants under 5 years of age, BMI z-scores specific for sex and age were calculated according to the growth curves published by WHO in $2006^{32}$ using ANTHRO 2005 software downloaded from the WHO website (http://www. who.int/childgrowth/software/en/). At the 6-year and 11-year follow-ups BMI were standardised by age and sex using the WHO 2007 growth reference. ${ }^{33}$

\section{Potential confounders}

Potential confounding variables-measured in the perinatal study-were family monthly income (Brazilian currency), maternal education (years of formal schooling), self-reported skin colour (white, brown, black), age (years), parity (number of previous viable pregnancies), smoking during pregnancy (yes/no) and pre-gestational BMI (calculated as $\mathrm{kg} / \mathrm{m}^{2}$ and categorised as normal weight when $\leq 24.9 \mathrm{~kg} / \mathrm{m}^{2}$, overweight $(25.0-$ $\left.29.9 \mathrm{~kg} / \mathrm{m}^{2}\right)$ and obesity $\left(\geq 30.0 \mathrm{~kg} / \mathrm{m}^{2}\right)$. Missing data were observed for maternal schooling and skin colour $(n=41)$ for each one of these variables and maternal BMI $(\mathrm{n}=335)$.

Given the major extent in miscegenation between Europeans, afrodescendants and (to a lesser extent) Native Americans, the official Brazilian classification for ethnicity relies on self-assessed skin colour. For this reason, skin colour was used rather than ethnicity.

Maternal weight before pregnancy was based on recall. Maternal height measurement was performed by trained team members during the 3-month follow-up visit, using an aluminium stadiometre with $1 \mathrm{~mm}$ precision.

Maternal smoking was evaluated retrospectively at the time of delivery. Women who had smoked at least one cigarette a day in any trimester of the pregnancy were classified as regular smokers.

These potential confounders were chosen a priori, consistent with published literature on maternal conditions during pregnancy and offspring BMI. ${ }^{34}{ }^{35}$ Family income, maternal schooling and maternal age were included in the models as continuous.

\section{Anthropometry training}

On each follow-up, anthropometry training sessions for measurement of cohort participants and their mothers were undertaken until all technical errors were within the acceptable limits previously set. During the standardisation process, which took place about 2 weeks before starting fieldwork, intra/inter-observer technical errors of measurements were calculated and compared with the measurements obtained by the anthropometry supervisor (gold standard). The number of anthropometrits in each follow-up varied between 5 and 10 . The measurements were standardised according to the Habitch criteria. ${ }^{36}$

\section{Statistical analysis}

The prevalence of GDM with 95\% CI was first calculated. We used $\chi^{2}$ test to compare proportions and Student's t-test to compare means of binary variables and ANOVA in the comparison of means of categorical variables. Univariable and multiple linear regressions were performed to estimate, respectively, unadjusted and adjusted coefficients of the association between GDM and offspring BMI z-score, independently for each follow-up from birth to 11 years of age. We first adjusted for confounding variables except pre-gestational maternal BMI. Subsequently, pre-gestational maternal BMI was entered in the model. Analyses of BMI z-scores at birth were further adjusted for gestational age (GA) ${ }^{37}$ Interaction terms were included in the adjusted models to explore the role of the maternal pre-gestational BMI as a possible effect modifier on the association between GDM and offspring z-score BMI at each age. When a statistically significant interaction term was found, subsequent analyses were stratified. Statistical comparisons between categories were based on tests of heterogeneity.

In addition, in order to assess the longitudinal effect of GDM over changes of BMI through time, we used unadjusted and adjusted multilevel models, considering two levels: (1) child and (2) BMI z-score evaluated at each time point (birth, 3, 12, 24, 48 months and 6 and 11 years). Unstructured covariance matrix was considered and intraclass correlation coefficients (ICC) were estimated for unadjusted and adjusted models. The mixed command was used. We used STATA V.14.1 (StataCorp) for all the analyses.

\section{Patient and public involvement \\ No patients were involved in this study.}

\section{RESULTS}

Table 1 shows the proportions of cohort members located at each follow-up according to maternal characteristics at the perinatal phase. Regarding family income, the highest losses at each follow-up from 12 months to 11 years were observed for those children born to the poorest families (lowest income quintile) $(89.2 \%, 88.5 \%, 87.2 \%, 83.9 \%$ and $79.6 \%$ were located at 12 -month, 24 -month, 48 -month and at 6-year and 11-year follow-ups, respectively). At 12-month, 6-year and 11-year waves, follow-up rates were lower among those whose mothers were less educated $(0$ to 4 years of formal schooling) $(90.0 \%, 84.3 \%$ and $80.6 \%$, respectively), and only at 6 and 11 years, follow-up rates were lower in those whose mothers had two children or more $(86.0 \%$ and $82.4 \%$, respectively) and among those whose mothers had not reported GDM $(87.8 \%$ and $84.1 \%$, respectively). According to maternal pre-gestational BMI, higher follow-up losses were observed in the normal BMI category at 3-month, 12-month and at 11-year phases; however, about $80 \%$ of subjects in any category of the baseline variable were traced. There were no differences in follow-up rates according to maternal 
Table 1 Characteristics of cohort members' mothers from single pregnancies and percentage located at each follow-up. Pelotas 2004 Birth Cohort

\begin{tabular}{|c|c|c|c|c|c|c|c|}
\hline \multirow[b]{2}{*}{ Characteristics (\%) } & \multicolumn{7}{|c|}{ Percentage located } \\
\hline & $\begin{array}{l}\text { Perinatal } \\
(n=4131)\end{array}$ & $\begin{array}{l}3 \text { months } \\
(n=3893)\end{array}$ & $\begin{array}{l}12 \text { months } \\
(\mathrm{n}=3815)\end{array}$ & $\begin{array}{l}24 \text { months } \\
(\mathrm{n}=3777)\end{array}$ & $\begin{array}{l}48 \text { months } \\
(n=3709)\end{array}$ & $\begin{array}{l}6 \text { years } \\
(n=3635)\end{array}$ & $\begin{array}{l}11 \text { years } \\
(n=3481)\end{array}$ \\
\hline \multicolumn{2}{|c|}{ Family income (quintiles) } & $P=0.086$ & $P<0.001$ & $P<0.001$ & $P=0.001$ & $P<0.001$ & $P<0.001$ \\
\hline 1 (poorest) & $845(20.4)$ & 93.1 & 89.2 & 88.5 & 87.2 & 83.9 & 79.6 \\
\hline 2 & 840 (20.3) & 93.0 & 90.5 & 90.1 & 89.1 & 88.2 & 84.1 \\
\hline 3 & $802(19.4)$ & 95.0 & 94.8 & 93.8 & 92.3 & 89.4 & 86.8 \\
\hline 4 & $846(20.4)$ & 95.6 & 94.7 & 93.4 & 92.2 & 90.7 & 87.8 \\
\hline 5 (richest) & $812(19.6)$ & 94.6 & 92.7 & 91.5 & 88.2 & 87.8 & 83.1 \\
\hline \multicolumn{2}{|l|}{ Skin colour } & $P=0.464$ & $P=0.301$ & $P=0.754$ & $P=0.540$ & $P=0.065$ & $P=0.636$ \\
\hline White & $3028(73.0)$ & 94.4 & 92.7 & 91.6 & 90.0 & 88.6 & 84.5 \\
\hline Brown & $289(7.0)$ & 94.8 & 91.7 & 90.3 & 87.8 & 84.1 & 82.4 \\
\hline Black & $828(20.0)$ & 93.4 & 91.2 & 91.3 & 89.7 & 87.0 & 84.2 \\
\hline \multicolumn{2}{|l|}{ Schooling (years) } & $P=0.052$ & $P=0.006$ & $P=0.143$ & $P=0.053$ & $P=0.005$ & $P=0.014$ \\
\hline $0-4$ & 638 (15.6) & 93.0 & 90.1 & 90.0 & 87.2 & 84.3 & 80.6 \\
\hline $5-8$ & $1691(41.2)$ & 93.9 & 91.8 & 91.1 & 90.4 & 89.0 & 84.6 \\
\hline 9 or more & 1775 (43.3) & 95.3 & 93.8 & 92.3 & 90.3 & 88.5 & 85.4 \\
\hline \multicolumn{2}{|l|}{ Age (years) } & $P=0.655$ & $P=0.069$ & $P=0.197$ & $P=0.210$ & $P=0.175$ & $P=0.103$ \\
\hline$<20$ & 799 (19.1) & 93.7 & 90.5 & 90.1 & 88.8 & 88.4 & 83.8 \\
\hline 20-35 & 2918 (70.4) & 94.3 & 92.6 & 91.5 & 89.7 & 87.5 & 83.9 \\
\hline$>35$ & $434(10.5)$ & 94.9 & 93.8 & 93.1 & 91.9 & 90.6 & 87.8 \\
\hline \multicolumn{2}{|l|}{ Parity } & $P=0.518$ & $P=0.861$ & $P=0.510$ & $P=0.380$ & $P=0.018$ & $P=0.020$ \\
\hline 0 & $1643(39.7)$ & 93.8 & 92.5 & 91.7 & 89.4 & 89.2 & 84.4 \\
\hline 1 & $1084(26.2)$ & 94.8 & 92.0 & 92.0 & 90.9 & 88.7 & 86.5 \\
\hline 2 or more & 1417 (34.2) & 94.3 & 92.5 & 90.8 & 89.3 & 86.0 & 82.4 \\
\hline \multicolumn{2}{|c|}{ Pre-gestational BMI categories } & $P=0.003$ & $P=0.004$ & $P=0.050$ & $P=0.153$ & $P=0.106$ & $P=0.010$ \\
\hline Normal & $2529(66.4)$ & 95.5 & 93.0 & 92.2 & 90.6 & 88.4 & 84.8 \\
\hline Overweight & $870(24.0)$ & 97.8 & 96.0 & 94.1 & 92.2 & 90.7 & 88.3 \\
\hline Obesity & $409(10.7)$ & 97.6 & 94.4 & 94.9 & 92.9 & 90.7 & 87.3 \\
\hline \multicolumn{2}{|c|}{ Gestational diabetes mellitus } & $P=0.352$ & $P=0.642$ & $P=0.660$ & $P=0.193$ & $P=0.017$ & $P=0.032$ \\
\hline No & $4020(97.4)$ & 94.2 & 92.3 & 91.4 & 89.7 & 87.8 & 84.1 \\
\hline Yes & $108(2.6)$ & 96.3 & 93.5 & 92.6 & 93.5 & 95.4 & 91.7 \\
\hline
\end{tabular}

$N$ of each follow-up excludes gemelar births $(n=84)$ and mothers who already had diabetes mellitus before the pregnancy $(n=14)$. $\mathrm{P}$-value, $\mathrm{X}^{2}$ test comparing the distribution of characteristics of mothers at perinatal and at each subsequent follow-up. BMI, body mass index.

skin colour or age at any wave of the study. Means of family income (Brazilian currency), maternal schooling (years) and mother's age (years) of participants included in this study at each follow-up are presented in online supplementary table 1 .

Prevalence of self-reported GDM-pregnancy onsetaccessed at the perinatal follow-up was $2.6 \%(95 \%$ CI $2.1 \%$ to $3.1 \%)(\mathrm{n}=108)$. The means and SD of offspring BMI z-score at birth, 3, 12, 24, 48 months and at 6 and 11 years were 0.10 (1.12), -0.47 (1.10), 0.59 (1.10), 0.59 (1.08), 0.78 (1.32), 0.70 (1.43) and 0.75 (1.41), respectively.
The association between GDM and offspring BMI z-score at every follow-up is shown in table 2. Unadjusted analyses showed that BMI z-score at birth and at ages 6 and 11 years old were higher among offspring born to mothers who had GDM compared with those whose mothers had not $(\beta=0.53,95 \%$ CI 0.33 to $0.74 ; \beta=0.30,95 \%$ CI 0.01 to 0.59 and $\beta=0.42,95 \%$ CI 0.13 to 0.71 , respectively). Unadjusted means (SD) according to maternal GDM status are shown in online supplementary table 2 . After adjustment for potential confounders (family income and maternal education, age, skin colour, parity and smoking 
Table 2 Unadjusted and adjusted association between GDM and offspring BMI z-score at birth, 3, 12, 24, 48 months and 6 and 11 years of age. Pelotas 2004 Birth Cohort

\begin{tabular}{|c|c|c|c|c|c|c|c|}
\hline \multirow{2}{*}{$\begin{array}{l}\text { BMI } \\
\text { z-score }\end{array}$} & \multicolumn{2}{|l|}{ Unadjusted } & \multicolumn{2}{|l|}{ Adjusted (model 1)* } & \multicolumn{2}{|l|}{ Adjusted (model 2)† } & \multirow{2}{*}{$\begin{array}{l}\text { Interaction } \\
\text { P valueף }\end{array}$} \\
\hline & $\beta(95 \% \mathrm{Cl})$ & P value§ & $\beta(95 \% \mathrm{Cl})$ & P value§ & $\beta(95 \% \mathrm{Cl})$ & P value§ & \\
\hline Birth** & 0.53 (0.33 to 0.74$)$ & $<0.001$ & 0.44 (0.24 to 0.63$)$ & $<0.001$ & 0.35 (0.14 to 0.55$)$ & 0.001 & 0.007 \\
\hline 12 months & $-0.06(-0.27$ to 0.15$)$ & 0.573 & $-0.06(-0.28$ to 0.16$)$ & 0.584 & $-0.07(-0.29$ to 0.16$)$ & 0.561 & 0.108 \\
\hline 24 months & $-0.13(-0.34$ to 0.09$)$ & 0.247 & $-0.15(-0.38$ to 0.06$)$ & 0.162 & $-0.20(-0.43$ to 0.02$)$ & 0.074 & 0.788 \\
\hline 6 years & 0.30 (0.01 to 0.59$)$ & 0.046 & $0.21(-0.08$ to 0.50$)$ & 0.159 & $0.10(-0.19$ to 0.38$)$ & 0.520 & 0.325 \\
\hline 11 years & $0.42(0.13$ to 0.71$)$ & 0.004 & 0.35 (0.07 to 0.64$)$ & 0.015 & $0.19(-0.10$ to 0.47$)$ & 0.197 & 0.569 \\
\hline
\end{tabular}

*Model 1: adjusted for family income, maternal education and mother's age at birth, mother's skin colour, parity and maternal smoking during pregnancy and sex of the child.

†Model 2: adjusted for model 1+maternal pre-gestational BMI.

łInteraction between maternal pre-gestational BMI and GDM in adjusted model 2.

$\S F$ test for the association between GDM and BMI z-scores (unadjusted and adjusted for covariates at models 1 and 2).

१F test for interaction between GDM and maternal pre-gestational BMI.

${ }^{* *} \mathrm{BMI}$ z-score at birth was adjusted for gestational age.

BMI, body mass index; GDM, gestational diabetes mellitus.

in pregnancy), BMI z-score at birth and at age 11 years remained associated with GDM ( $\beta=0.44,95 \%$ CI 0.24 to 0.63 and $\beta=0.35,95 \%$ CI 0.07 to 0.64 , respectively). When maternal pre-gestational BMI was included in the analyses (adjusted model 2), only the association between GDM and BMI z-score at birth remained significant $(\beta=0.35$, $95 \%$ CI 0.14 to 0.55 ).

In general, adjusted model 1 resulted in modest attenuation of the coefficients compared with the unadjusted model. However, the inclusion of maternal pre-gestational BMI (adjusted model 2) resulted in more marked attenuation. For example, at age 11, regression coefficients decreased $17 \%$ from unadjusted to adjusted model 1 , and $46 \%$ from adjusted model 1 to adjusted model 2.

Statistical evidence of effect modification between maternal pre-gestational BMI and GDM over the offspring BMI z-score was found only at birth (p-value for the interaction term=0.007) (table 2). Presence of effect modification indicates that the association between GDM and offspring BMI z-score differed according to maternal pre-gestational BMI categories. Subsequent stratified analyses by maternal pre-pregnancy BMI (table 3), showed that the association between GDM and BMI z-score at birth disappeared when mothers had normal pre-gestational BMI. However, the association between GDM and BMI z-score at birth strengthened among newborns of mothers from the overweight and obese pre-gestational BMI groups ( $\beta=0.72,95 \%$ CI 0.30 to 1.14 and $\beta=0.61,95 \%$ CI 0.20 to 1.01 , respectively). When overweight and obese categories were grouped into one, the BMI z-score at birth was 0.74 (95\% CI 0.45 to 1.02$)$ higher in offspring of mothers with GDM compared with those born to mothers without GDM (data not shown). Unadjusted means (SD) BMI z-score at birth, 3, 12, 24 and 48 months and 6 and 11 years of age according to maternal pre-gestational BMI categories are shown in online supplementary table 3 .

Table 4 shows the multilevel models of the association between GDM and offspring BMI z-score analysed as repeated measurements for participants at each time point. No association was observed in unadjusted and adjusted models 1 and $2(\beta=0.13,95 \%$ CI -0.04 to 0.29 ;

Table 3 Association between GDM and offspring BMI z-score at birth*, stratified by maternal pre-pregnancy BMI. Pelotas 2004 Birth Cohort

\begin{tabular}{|c|c|c|c|c|}
\hline \multirow[b]{2}{*}{ Maternal pre-gestational BMI } & \multicolumn{4}{|c|}{ Association between GDM and BMI z-score at birth } \\
\hline & Unadjusted $\beta(95 \% \mathrm{Cl})$ & $\mathbf{P}$ value & Adjusted $\beta$ ( $(95 \% \mathrm{Cl})$ & P valuet \\
\hline Normal $\left(\leq 24.9 \mathrm{~kg} / \mathrm{m}^{2}\right)(\mathrm{n}=2486)$ & $0.12(-0.18$ to 0.42$)$ & 0.430 & $0.03(-0.27$ to 0.32$)$ & 0.849 \\
\hline Overweight $\left(25.0-29.9 \mathrm{~kg} / \mathrm{m}^{2}\right)(\mathrm{n}=848)$ & $0.74(0.32$ to 1.16$)$ & 0.001 & $0.72(0.30$ to 1.14$)$ & 0.001 \\
\hline Obese $\left(\geq 30.0 \mathrm{~kg} / \mathrm{m}^{2}\right)(n=399)$ & 0.64 (0.24 to 1.03$)$ & 0.002 & $0.61(0.20$ to 1.01$)$ & 0.003 \\
\hline
\end{tabular}

Data are $\beta(95 \% \mathrm{Cl})$.

${ }^{*} \mathrm{BMI}$ z-score at birth was adjusted for gestational age.

†F test for the association between GDM and BMI z-scores in each pre-gestational BMI category .

¥Family income and maternal education, skin colour, parity and age at delivery.

BMI, body mass index; GDM, gestational diabetes mellitus. 
Table 4 Multilevel models for the association between GDM and longitudinal data on offspring BMI z-scores. Pelotas 2004 Birth Cohort

\begin{tabular}{|c|c|c|c|c|c|c|c|}
\hline & \multirow{2}{*}{$\begin{array}{l}\text { Null model } \\
\beta(95 \% \mathrm{Cl})\end{array}$} & \multicolumn{2}{|l|}{ Unadjusted } & \multicolumn{2}{|l|}{ Adjusted (model 1)* } & \multicolumn{2}{|l|}{ Adjusted (model 2)† } \\
\hline & & $\beta(95 \% \mathrm{Cl})$ & P valueł & $\beta(95 \% \mathrm{Cl})$ & $P$ value & $\beta(95 \% \mathrm{Cl})$ & P value \\
\hline BMI z-score & - & $0.13(-0.04$ to 0.29$)$ & 0.142 & $0.07(-0.10$ to 0.24$)$ & 0.413 & $-0.01(-0.17$ to 0.17$)$ & 0.967 \\
\hline
\end{tabular}

*Model 1: adjusted for family income, maternal education and mother's age at birth, mother's skin colour, parity and maternal smoking during pregnancy and sex of the child.

†Model 2: adjusted for model 1+maternal pre-gestational BMI.

fLikelihood ratio test for the association between GDM and BMI z-score at different time points (birth, 3, 12, 24, 48 months and 6 and 11 years of age) using multilevel analyses (unadjusted and adjusted for covariates at models 1 and 2).

BMI, body mass index; GDM, gestational diabetes mellitus; ICC, intraclass correlation coefficients.

$\beta=0.07,95 \%$ CI -0.10 to 0.24 and $\beta=-0.01,95 \% \mathrm{CI}$ -0.17 to 0.17 , respectively).

\section{DISCUSSION}

In this study, GDM was found to be associated with offspring BMI z-scores at birth and at ages 6 and 11 years; however, the association at ages 6 and 11 years disappeared after adjustment for confounders. In addition, we found that maternal pre-gestational BMI modified the association between GDM and offspring BMI at birth. Higher means of BMI z-score at birth were observed only in those children whose mothers had GDM and were also overweight or obese before the gestation.

Thus, our results suggest that the combination of both conditions together increases the risk of higher BMI at birth, which, in turn, is a risk factor for developing obesity at later ages. ${ }^{38}$ This is of great concern for the clinical practice and public health policies, since the majority of mothers with GDM are obese, and a significant proportion of those who are obese have GDM. ${ }^{39}$ A meta-analysis of observational cohort studies about maternal obesity and the risk of GDM showed that, compared with normalweight pregnant women, overweight and obese women had 2.14 (95\% CI 1.82 to 2.53 ) and 3.56 (95\% CI 3.05 to 4.21 ) increased odds of developing GDM, respectively. ${ }^{40}$ In our study we found that the mean maternal pre-gestational BMI was higher in mothers who had diabetes during pregnancy than in mothers who had not (26.9 vs $\left.24.0 \mathrm{~kg} / \mathrm{m}^{2} ; \mathrm{p}<0.001\right)$. Moreover, the prevalence of GDM among mothers with normal pre-pregnancy BMI was $1.9 \%$, whereas the prevalence among overweight and obese mothers was $3.0 \%$ and $8.0 \%$, respectively $(p=0.001)$.

Relatively few studies that examined the association between GDM and offspring BMI have considered the potential role of the maternal pre-gestational BMI as a confounder in this association. ${ }^{27}{ }^{41}$ Consistent with our results at age 11 years, analyses carried out in a large pregnancy and birth cohort in England Avon Longitudinal Study of Parents and Children (ALSPAC) revealed that positive associations between GDM and higher offspring BMI z-score in childhood attenuated towards the null with the inclusion of pre-gestational BMI in the models.
At age 9-11 years, BMI z-score mean differences between children exposed to and non-exposed to GDM were 0.33 (95\% CI 0.01 to 0.64 ) (adjusted for GA, maternal age, social class, parity, smoking during pregnancy and mode of delivery) and 0.01 (95\% CI -0.30 to 0.63 ) with further adjustment for pre-gestational BMI. ${ }^{18}$ Results from the BetaGene Cohort also are in concordance with our findings. In this prebirth cohort of self-reported Mexican American, the association between GDM and offspring BMI z-scores between the ages of 5 and 16 years old (mean difference in BMI z-score between those exposed to GDM and those not exposed of $0.66 ; 95 \% \mathrm{CI} 0.01$ to 1.32 ) was not longer apparent when maternal pre-gestational BMI and weight gain during pregnancy were included in the analyses (mean difference $0.63 ; 95 \%$ CI -0.02 to 1.28 ) ${ }^{21}$ Project Viva, a prospective prebirth cohort, showed that GDM was not associated with offspring BMI $z$-score at age 3 years in adjusted models for maternal age, education, ethnicity, smoking history, BMI, parity and paternal BMI (mean difference equal to $-0.08,95 \%$ CI -0.37 to 0.22$){ }^{16}$

These results support the hypothesis that maternal BMI plays a confounding role in the association between GDM and offspring BMI. ${ }^{27} 4142$ Maternal pre-gestational BMI is a well-known risk factor for GDM, ${ }^{39} 40$ but its relationship with offspring BMI may well operate through several pathways. ${ }^{8}$ Even though it is possible that maternal obesity increases the risk of developing GDM, which, in turn, could lead to higher offspring BMI. Other explanations include epigenetic modification (developmental overnutrition theory), post-natal shared environmental influences (eg, dietary behaviours) and genetic inheritance. ${ }^{8}$ Future studies with emphasis on casuality approaches are needed to assess the contribution of GDM and pre-gestational BMI on the offspring long-term health conditions.

Our study has three major strengths. First, the repeated anthropometric measures from a long follow-up, which allowed us to assess the association between GDM and BMI $z$-score at several points from birth until early adolescence (across infancy and childhood). Second, the inclusion of the maternal pre-gestational BMI in the analyses, which enabled us to identify the role of this variable as a confounder or effect modifier of the association 
between GDM and offspring BMI z-score. Furthermore, several relevant confounders previously recognised in published literature were controlled for in our analyses, thus reducing the possibility of biased estimates due to residual confounding. Third, the population-based large sample size, which makes our findings generalisable to larger populations with similar confounding structure as our study population, in terms of demographic, socioeconomic and behavioral characteristics. Our study sample provides complete coverage of the Pelotas city population, which has similar characteristics of other Brazilian cities (eg, maternal skin colour, education and obesity rates) ${ }^{43}$ Other strengths are the high rates of follow-ups (at least $80 \%$ of subjects in any category of the baseline variables were available for the current analyses), and no losses in our main exposure (GDM), diminishing the possibility of selection bias. The use of standardised units in our outcomes (z-scores) is another strength that will facilitate future meta-analyses in this area. Furthermore, we were able to distinguish between GDM and existing diabetes. This is important because women with diagnosis of diabetes (type 1 or type 2 ) before pregnancy possibly represent a group experiencing a distinctive spectrum of the disease.

On the other hand, the lack of data on glycaemic control during pregnancy is a limitation, as the treatment of GDM may be an important determinant of offspring outcomes. ${ }^{744}$ Other limitations of our data are the assessment of GDM and maternal weight based on self-reporting, which could lead to misclassification. However, validation studies for both-self-reported GDM and pre-gestational weight-suggested that the self-report is a valid source of information on these sujects among our population. Regarding GDM, a population-based study evaluating the agreement between self-reported GDM and prenatal care medical records (gold standard) in the city of Pelotas concluded that self-reported GDM is valid information for this population. ${ }^{31}$ The study showed a sensibility of $72.9 \%$. A higher sensibility would increase the power of the study to identify associations; however, the direction of the coefficient would not be affected. Validity of self-reported weight was assessed in adults from Porto Alegre, southern Brazil. The mean difference between measured and self-reported weight in women was $0.29 \mathrm{~kg}$, and the correlation was high $(\mathrm{r}=0.95)$. The authors concluded that the validity of reported weight is acceptable for epidemiological study surveys in similar settings. ${ }^{45}$ A study comparing self-reported versus measured BMI in a pregnancy cohort (Pregnancy Outcomes, Maternal and Infant Study) in Peru observed a mean difference of $0.27 \mathrm{~kg}$ between weight measured at the first antenatal visit and self-reported weight. This suggests that, in Peru and other low/middle- income countries, the high concordance between self-reported and measured weight would allow for adequate BMI category classification. ${ }^{46}$ Finally, we had no information on potential confounders as family history of diabetes. Also, there exists the possibility of residual confounding due to crude categorisation of smoking.
However, the inclusion of other confounders or a more complete information on smoking would attenuate the coefficients towards the null more than the attenuations already observed; thus, our findings would not change.

Summing up, our data showed that the concomitant exposure to GDM and maternal overweight or obesity lead to greater risk of higher BMI z-scores at birth. Furthermore, we observed that maternal pre-gestational BMI accounted for the association between GDM and offspring BMI z-score at 11 years. We conclude that diabetic control remains important, although strategies to prevent higher BMI means during childhood and early adolescence should include interventions targeted to the promotion of a healthy nutritional status (eg, normal $\mathrm{BMI})$ of women at reproductive age.

Acknowledgements We are grateful to all the children who took part in the Pelotas birth cohorts, and the Pelotas teams, including research scientists, interviewers, workers and volunteers.

Contributors RB and ISS designed the study. RB and CLdM performed the analysis and contributed to the interpretation of the results. AB, AM and ISS participated in the design and conduct of the original cohort studies as well as in interpreting results and critical reviewing of the manuscript. RB wrote the manuscript, and all authors contributed to and approved the final version.

Funding This article is based on data from the study 'Pelotas Birth Cohort, 2004' conducted by Postgraduate Program in Epidemiology at Universidade Federal de Pelotas, with the collaboration of the Brazilian Public Health Association (ABRASCO). From 2009 to 2013, the Wellcome Trust supported the 2004 birth cohort study (grant number 086974/Z/08/Z). The World Health Organization (process number 03014HNI), National Support Program for Centers of Excellence (PRONEX) (process number 04/0882.7), Brazilian National Research Council (CNPq) (process numbers 481,012-2009-5; 484,077-2010-4; 470,965-2010-0 and 481,141-2007-3), Brazilian Ministry of Health (process number 4589-04) and Children's Pastorate supported previous phases of the study. RB, AB, AM and ISS are supported by the CNPq. The study sponsors had no involvement in study design; the collection, analysis and interpretation of data; the writing of the report; nor in the decision to submit the paper for publication. No honorarium, grant or other form of payment was given to anyone to produce the manuscript.

Competing interests None declared.

Patient consent for publication Not required.

Ethics approval The study and its protocols were approved by the School of Medicine Ethics Committee of the Federal University of Pelotas. Mothers signed a consent form on behalf of them and their children at each follow-up, after being informed of the study objectives. At the 11-year follow-up, the cohort participants aged 11 years or more also signed an informed consent form.

Provenance and peer review Not commissioned; externally peer reviewed.

Data sharing statement Due to confidentiality restrictions related to the ethics approval for this study, no identifying information about participants may be released. Data set without identification used during the current study is available from the corresponding author on reasonable request.

Open access This is an open access article distributed in accordance with the Creative Commons Attribution Non Commercial (CC BY-NC 4.0) license, which permits others to distribute, remix, adapt, build upon this work non-commercially, and license their derivative works on different terms, provided the original work is properly cited, appropriate credit is given, any changes made indicated, and the use is non-commercial. See: http://creativecommons.org/licenses/by-nc/4.0/.

\section{REFERENCES}

1. World Health Organization. Definition, diagnosis and classification of diabetes mellitus and its complications: report of a WHO consultation. Part 1, Diagnosis and classification of diabetes mellitus, 1999. 
2. Hadden DR. Geographic, ethnic, and racial variations in the incidence of gestational diabetes mellitus. Diabetes 1985;34 Suppl 2(Suppl 2):8-12.

3. Dooley SL, Metzger BE, Cho N, et al. The influence of demographic and phenotypic heterogeneity on the prevalence of gestational diabetes mellitus. Int J Gynaecol Obstet 1991;35:13-18.

4. Hartling L, Dryden DM, Guthrie A, et al. Screening and diagnosing gestational diabetes mellitus. Evid Rep Technol Assess 2012:1-327.

5. Committee on Practice Bulletins--Obstetrics. Practice Bulletin No. 137: Gestational diabetes mellitus. Obstet Gynecol 2013;122(2 Pt 1):406-16.

6. Jones CW. Gestational diabetes and its impact on the neonate. Neonatal Netw 2001;20:17-23.

7. Metzger BE, Lowe LP, Dyer AR, et al. Hyperglycemia and adverse pregnancy outcomes. N Engl J Med 2008;358:1991-2002.

8. Lawlor DA. The Society for Social Medicine John Pemberton Lecture 2011. Developmental overnutrition--an old hypothesis with new importance? Int J Epidemiol 2013;42:7-29.

9. Fraser A, Lawlor DA. Long-term health outcomes in offspring born to women with diabetes in pregnancy. Curr Diab Rep 2014;14:489.

10. Ebbeling CB, Pawlak DB, Ludwig DS. Childhood obesity: publichealth crisis, common sense cure. Lancet 2002;360:473-82.

11. NCD Risk Factor Collaboration (NCD-RisC). Worldwide trends in body-mass index, underweight, overweight, and obesity from 1975 to 2016: a pooled analysis of 2416 population-based measurement studies in 128.9 million children, adolescents, and adults. Lancet 2017;390:2627-42

12. Lobstein T, Jackson-Leach R, Moodie ML, et al. Child and adolescent obesity: part of a bigger picture. Lancet 2015;385:2510-20.

13. UNICEF (United Nations Childrens's Fund), WHO (Wolrd Health Organization), Bank W. Levels and trends in child malnutrition: joint child malnutrition estimates, key findings of the 2017 edition, 2017.

14. Gillman MW, Rifas-Shiman S, Berkey CS, et al. birth weight, and adolescent obesity. Pediatrics 2003;111:e221-6.

15. Catalano PM, Farrell K, Thomas A, et al. Perinatal risk factors for childhood obesity and metabolic dysregulation. Am J Clin Nutr 2009;90:1303-13.

16. Wright CS, Rifas-Shiman SL, Rich-Edwards JW, et al. Intrauterine exposure to gestational diabetes, child adiposity, and blood pressure. Am J Hypertens 2009;22:215-20.

17. Krishnaveni GV, Veena SR, Hill JC, et al. Intrauterine exposure to maternal diabetes is associated with higher adiposity and insulin resistance and clustering of cardiovascular risk markers in Indian children. Diabetes Care 2010;33:402-4.

18. Lawlor DA, Fraser A, Lindsay RS, et al. Association of existing diabetes, gestational diabetes and glycosuria in pregnancy with macrosomia and offspring body mass index, waist and fat mass in later childhood: findings from a prospective pregnancy cohort. Diabetologia 2010;53:89-97.

19. Lindsay RS, Nelson SM, Walker JD, et al. Programming of adiposity in offspring of mothers with type 1 diabetes at age 7 years. Diabetes Care 2010;33:1080-5.

20. Crume TL, Ogden L, West NA, et al. Association of exposure to diabetes in utero with adiposity and fat distribution in a multiethnic population of youth: the Exploring Perinatal Outcomes among Children (EPOCH) Study. Diabetologia 2011;54:87-92.

21. Page KA, Romero A, Buchanan TA, et al. Gestational diabetes mellitus, maternal obesity, and adiposity in offspring. J Pediatr 2014;164:807-10.

22. Whitaker RC, Pepe MS, Seidel KD, et al. Gestational diabetes and the risk of offspring obesity. Pediatrics 1998;101:E9.

23. Manderson JG, Mullan B, Patterson CC, et al. Cardiovascular and metabolic abnormalities in the offspring of diabetic pregnancy. Diabetologia 2002;45:991-6.

24. Hunter WA, Cundy T, Rabone D, et al. Insulin sensitivity in the offspring of women with type 1 and type 2 diabetes. Diabetes Care 2004;27:1148-52.
25. Pettitt DJ, Nelson RG, Saad MF, et al. Diabetes and obesity in the offspring of Pima Indian women with diabetes during pregnancy. Diabetes Care 1993;16:310-4

26. Tam WH, Ma RC, Yang X, et al. Glucose intolerance and cardiometabolic risk in children exposed to maternal gestational diabetes mellitus in utero. Pediatrics 2008;122:1229-34.

27. Philipps LH, Santhakumaran S, Gale C, et al. The diabetic pregnancy and offspring BMI in childhood: a systematic review and metaanalysis. Diabetologia 2011;54:1957-66.

28. Santos IS, Barros AJ, Matijasevich A, et al. Cohort profile: the 2004 Pelotas (Brazil) birth cohort study. Int J Epidemiol 2011;40:1461-8.

29. Santos IS, Barros AJ, Matijasevich A, et al. Cohort profile update: 2004 Pelotas (Brazil) Birth Cohort Study. Body composition, mental health and genetic assessment at the 6 years follow-up. Int $J$ Epidemiol 2014;43:1437-1437f.

30. Barros AJ, da Silva dos Santos I, Victora CG, et al. [The 2004 Pelotas birth cohort: methods and description]. Rev Saude Publica 2006;40:402-13

31. Dode MA, Santos IS. [Validity of self-reported gestational diabetes mellitus in the immediate postpartum]. Cad Saude Publica 2009;25:251-8

32. World Health Organization. WHO Multicentre Growth Reference Study Group: WHO child growth standards: length/height-for-age, weight-for-age, weight-for-length, weight-for-height and body mass index-for-age: Methods and development. Geneva: WHO 2007.

33. de Onis M, Onyango AW, Borghi E, et al. Development of a WHO growth reference for school-aged children and adolescents. Bull World Health Organ 2007;85:660-7.

34. Ehrenthal DB, Maiden K, Rao A, et al. Independent relation of maternal prenatal factors to early childhood obesity in the offspring. Obstet Gynecol 2013;121:115-21.

35. Ziyab AH, Karmaus W, Kurukulaaratchy RJ, et al. Developmental trajectories of Body Mass Index from infancy to 18 years of age: prenatal determinants and health consequences. J Epidemiol Community Health 2014;68:934-41.

36. Habicht JP. [Standardization of quantitative epidemiological methods in the field]. Bol Oficina Sanit Panam 1974;76:375.

37. Perumal N, Roth DE, Perdrizet J, et al. Effect of correcting for gestational age at birth on population prevalence of early childhood undernutrition. Emerg Themes Epidemiol 2018;15:3.

38. Schellong K, Schulz S, Harder T, et al. Birth weight and long-term overweight risk: systematic review and a meta-analysis including 643,902 persons from 66 studies and 26 countries globally. PLoS One 2012;7:e47776.

39. Simmons D. Diabetes and obesity in pregnancy. Best Pract Res Clin Obstet Gynaecol 2011;25:25-36.

40. Chu SY, Callaghan WM, Kim SY, et al. Maternal obesity and risk of gestational diabetes mellitus. Diabetes Care 2007;30:2070-6.

41. Kim SY, England JL, Sharma JA, et al. Gestational diabetes mellitus and risk of childhood overweight and obesity in offspring: a systematic review. Exp Diabetes Res 2011;2011:1-9.

42. Donovan LE, Cundy T. Does exposure to hyperglycaemia in utero increase the risk of obesity and diabetes in the offspring? A critical reappraisal. Diabet Med 2015;32:295-304.

43. IBGE. Insituto Brasileiro de Geografia e Estatística. www.ibge.gov.br.

44. Hillier TA, Pedula KL, Schmidt MM, et al. Childhood obesity and metabolic imprinting: the ongoing effects of maternal hyperglycemia. Diabetes Care 2007;30:2287-92.

45. Schmidt MI, Duncan BB, Tavares M, et al. Validity of self-reported weight--a study of urban Brazilian adults. Rev Saude Publica 1993;27:271-6.

46. Natamba BK, Sanchez SE, Gelaye B, et al. Concordance between self-reported pre-pregnancy body mass index (BMI) and BMI measured at the first prenatal study contact. BMC Pregnancy Childbirth 2016;16:187. 